\title{
Transferência de cuidados: processo de alta dos usuários de um centro de atenção psicossocial
}

Ariane da Cruz Guedes ${ }^{1}$, Agnes Olschowsky ${ }^{2}$, Luciane Prado Kantorski ${ }^{3}$, Milena Hohmann Antonacci ${ }^{4}$

\footnotetext{
${ }^{1}$ Enfermeira, Doutora em Enfermagem. Professora Adjunta da Faculdade de Enfermagem da Universidade Federal de Pelotas. Pelotas, RS, Brasil. E-mail: arianecguedes@gmail.com.

${ }^{2}$ Enfermeira, Doutora em Enfermagem Psiquiátrica. Professora Titular da Escola de Enfermagem da Universidade Federal do Rio Grande do Sul. Porto Alegre, RS, Brasil. E-mail: agnes@enf.ufrgs.br.

${ }^{3}$ Enfermeira, Doutora em Enfermagem. Professora Associada da Faculdade de Enfermagem da Universidade Federal de Pelotas. Pelotas, RS, Brasil. E-mail: kantorski@uol.com.br.

${ }^{4}$ Enfermeira, Doutora em Enfermagem Psiquiátrica. Professora do Colegiado de Enfermagem do Instituto Federal do Paraná Campus Palmas. Palmas, PR, Brasil. E-mail: mhantonacci@gmail.com.
}

Recebido: 23/10/2016.

Aceito: 31/08/2017.

Publicado: 01/12/2017.

\section{Como citar esse artigo:}

Guedes AC, Olschowsky A, Kantorski LP, Antonacci MH. Transferência de cuidados: processo de alta dos usuários de um centro de atenção psicossocial. Rev. Eletr. Enf. [Internet]. 2017 [acesso em: ____;19:a42. Disponível em: http://dx.doi.org/10.5216/ree.v19.43794.

\section{RESUMO}

A alta, em serviços de saúde mental, tem conotação de reabilitação psicossocial e articulação com os dispositivos da rede para promover a atenção continuada no território. Objetivou-se avaliar o processo de alta dos usuários de Centros de Atenção Psicossocial (CAPS). Pesquisa qualitativa e avaliativa, pautada na Avaliação de Quarta Geração. Realizada em um CAPS II de Santa Catarina, com 17 trabalhadores, 14 familiares e cinco usuários, em 2014. Utilizou-se o Método Comparativo Constante para análise dos dados. Compreende-se que o processo de alta é uma importante estratégia de reabilitação psicossocial, promovendo o cuidado no território de vida dos usuários. Este estudo põe em questão a cronificação nos CAPS, pois esses não devem ser serviços que mantenham os usuários em acompanhamento por longo período, mas ser transitórios, possibilitando o fortalecimento do cuidado em outros pontos da rede, estreitando vínculo com a atenção primária e com os demais equipamentos sociais do território.

Descritores: Alta do Paciente; Saúde Mental; Avaliação em Saúde; Serviços de Saúde Mental; Enfermagem Psiquiátrica.

\section{INTRODUÇÃO}

A temática da alta em saúde mental acompanha as modificações no modelo assistencial. Sua definição ainda está fortemente atrelada ao modelo hospitalar e ao seu ideal de cura. Contudo, a alta, neste estudo, se vincula ao contexto da atenção psicossocial, a qual a desvincula do sentido biológico, o qual remete à cura da patologia psiquiátrica. Aqui, ela tem conotação de reabilitação psicossocial, de produção de vida e de articulação com os diversos dispositivos da rede de saúde para promover a atenção continuada do usuário no território. De acordo com alguns estudos ${ }^{(1-5)}$ um dos pontos fundamentais para ao avanço no processo de 
alta é a presença de uma rede flexível, ágil e resolutiva, que dê suporte ao usuário no momento em que ele recebe alta. Portanto, é importante que ocorra o planejamento do processo de alta, de modo que o sujeito possa ter acesso à rede de acordo com sua necessidade específica, sendo este o ponto fundamental para a desinstitucionalização(5).

Neste sentido, a Comissão de Saúde Mental da Nova Zelândia apontou algumas estratégias para o desenvolvimento dos serviços de saúde mental, entre elas está a melhoria dos fluxos entre os serviços primários e os especializados, sendo necessário manter o foco na reabilitação, promovendo a integração na rede, fornecendo a provisão de serviços de maneira eficiente. Essas ações devem estar presentes em toda a rede, e não apenas em um serviço. Para isso, as equipes devem promover a alta do usuário dos serviços especializados, fortalecendo a construção de parcerias e relacionamentos em todos os pontos da rede a fim de assegurar uma transição efetiva ao sujeito, uma vez que usuários que não conseguem se vincular às equipes as quais são transferidos, têm maior dificuldade de adesão ao tratamento após a alta ${ }^{(6-8)}$.

Neste sentido, é importante a promoção e o reforço do vínculo entre as equipes de modo a ampliar acesso, diálogo e responsabilização pelas demandas de saúde mental. No momento em que a rede estiver articulada, com atenção qualificada em todos os seus pontos, tanto os profissionais quanto os usuários, estarão mais seguros para a promoção da alta, possibilitando que os indivíduos com transtorno psíquico sejam cuidados em seu território de vida, rompendo o estigma de que a atenção em saúde mental acontece apenas em serviços especializados.

É necessário que o usuário seja esclarecido quanto às oportunidades e benefícios que a alta lhe proporcionará, possibilitando construir novos vínculos com pessoas e serviços, ampliando assim sua rede social. Práticas voltadas à autonomia dos usuários devem estar presentes no cotidiano das equipes do CAPS, sendo que o recurso da alta contribui para que o usuário retome sua vida de maneira independente.

Tendo em vista que o fenômeno do processo de alta, no contexto da atenção psicossocial, precisa ser compreendido e aprofundado, este estudo objetivou avaliar o processo de alta dos usuários de um Centro de Atenção Psicossocial.

\section{MÉTODOS}

Pesquisa qualitativa de caráter avaliativo, do tipo estudo de caso, sendo um recorte da pesquisa de Avaliação dos Centros de Atenção Psicossocial da Região Sul do Brasil, em sua primeira e segunda edição (CAPSUL I I II) $^{(9-10)}$.

O presente estudo teve como referencial metodológico a avaliação de quarta geração, a qual pode ser definida como um modelo de avaliação em que as questões, reivindicações e preocupações dos grupos de interesse sirvam de enfoques organizacionais que são implementados segundo os preceitos metodológicos de investigação construtivista ${ }^{(11)}$.

A avaliação de quarta geração constitui-se em um fluxo de 12 passos para o desenvolvimento do processo avaliativo. 01 o passo é contratar, ou seja, formalizar o contrato com o participante ou instituição, 
nesse caso, com o CAPS. O 20 é organizar, visando à seleção e treinamento dos pesquisadores, organização da logística e trabalho de campo. No 3 o passo, busca-se identificar os grupos de interesses e também elaborar estratégias de busca dos participantes. 0 4ํㅡ passo é definido pela geração de construções conjuntas nos grupos, por meio da criação dos círculos hermenêuticos. Já, o 50 passo, objetiva ampliar as construções conjuntas dos interessados por meio de novas informações, podendo utilizar informações documentais e também fazer a interação entre entrevistas e dados de observações. O 60 passo busca selecionar as questões não resolvidas, separando-as como componentes de relato de caso. 0 70 visa priorizar as questões não resolvidas. 0 8o passo é a coleta de informações, com o objetivo de aumentar o nível de esclarecimento. 0 9o passo visa a preparar a agenda de negociação, e, o 10으, conduzir a negociação. 0 11 passo objetiva divulgar os resultados. E o 12 o passo é o da Reciclagem dos Dados, que objetiva reciclar o processo como um todo $^{(11)}$.

Neste estudo foi desenvolvida a Reciclagem dos Dados, a qual permite ao pesquisador lidar com questões trazidas por outros círculos, buscando aprofundar as informações, as questões e preocupações dos processos avaliativos anteriores ${ }^{(12)}$.

Inicialmente foram analisadas as duas edições da pesquisa CAPSUL, que deram subsídios para avaliar as questões que envolvem o processo de alta dos usuários do CAPS nos anos em que foram desenvolvidas, 2006 e 2011. Com base na análise dessas entrevistas de usuários, familiares e equipe do CAPS em questão, e ainda dos diários de campo que contemplam as observações do serviço, definiu-se e organizou-se a etapa de Reciclagem dos Dados.

O estudo foi desenvolvido no CAPS II de um município de grade porte do estado de Santa Catarina. A coleta de dados foi realizada por três pesquisadores, os quais realizaram observação do serviço, com registro em diários de campo, e conduziram Grupos de Reciclagem dos Dados (GRD). Essa etapa ocorreu em abril de 2014, por um período de duas semanas em campo. Na primeira foram realizadas observações de campo, com registros em diários de campo, totalizando 100 horas de observação; na segunda semana foram realizados os Grupos de Reciclagem dos Dados (GRD), com a introdução de questões referentes ao processo de alta identificadas nos bancos de dados CAPSUL I e II, e as observações da primeira semana da etapa de Reciclagem.

A fim de garantir o anonimato da identidade dos participantes, as informações serão identificadas de acordo com as fontes a seguir: Diário de Campo da Reciclagem dos Dados (exemplo: DC1RD), numerado de um a três, referenciando os três pesquisadores. E os Grupos de Reciclagem dos Dados identificados pela letra inicial do respectivo grupo de interesse (GRDU: usuários; GRDF: familiares; GRDT: trabalhadores).

Os GRD's tiveram duração de aproximadamente duas horas, com a participação de três pesquisadores, e de cada grupo de participantes: usuários, familiares e equipe, separadamente. Foram realizados em salas de oficinas e grupos do CAPS II. Os grupos foram conduzidos por um pesquisador, enquanto os outros realizavam registros em Diários de Campo. Foi utilizado o projetor multimídia e notebook para apresentar as questões introduzidas no grupo. Os encontros foram gravados em áudio, e posteriormente foram transcritos 
pelo pesquisador.

A análise dos dados foi realizada por meio do Método Comparativo Constante ${ }^{(13)}$, o qual permite que a análise dos dados se realize simultaneamente à coleta dos dados. Este apresenta duas etapas distintas: a primeira consiste na identificação das unidades de informação, as quais servem para a definição de categorias, e são obtidas por meio do material empírico coletado. Já, a segunda, consiste na categorização, cujo objetivo é agregar todas as unidades que têm relação com o mesmo conteúdo em categorias provisórias, objetivando a consistência interna das categorias.

Foram identificadas três categorias temáticas: Tempo de permanência no CAPS; Preparo para a alta e Continuidade do cuidado no território. E a categoria empírica central: Processo de alta. Para este estudo optou-se por apresentar um recorte dos resultados, no que se refere à compreensão da alta enquanto transferência de cuidados.

A etapa de Reciclagem dos Dados foi aprovada pelo Comitê de Ética em Pesquisa da Faculdade de Medicina da Universidade Federal de Pelotas (Parecer no 750.144, 2014). Foram respeitados os preceitos éticos em pesquisa com seres humanos, de acordo com a Resolução 466/2012 do Conselho Nacional de Saúde, do Ministério da Saúde ${ }^{(14)}$.

\section{RESULTADOS}

Por compreender que não há um consenso em saúde mental para a utilização do termo alta do usuário, optou-se em apresentar nesse estudo os resultados do enfoque na avaliação de usuários, familiares e profissionais do CAPS em questão, sobre a compreensão e utilização da alta enquanto "Transferência de cuidados". Essa concepção de alta foi indicada pelos participantes do estudo como a definição que mais se aproxima do que se quer traduzir do termo "alta" para o campo da saúde mental, nesse caso especificamente alta do CAPS.

Transferência de cuidados: uma nova compreensão sobre a alta

O termo "alta" do CAPS é questionado pelos profissionais:

Eu acho que a alta [...] acho que de todos nós aqui de saúde mental, é uma coisa que não existe, até por conta de uma doença que tem que ser tratada a longo prazo (GRDT).

A gente remete ao significado de alta quando a pessoa está curada (GRDT).

Surge, então, a definição proposta pelos trabalhadores do CAPS: transferência de cuidados:

Para mim, alta é a transferência de cuidados (GRDT).

Para mim também, para Unidade Básica de Saúde (UBS) e para a vida, para os dois (GRDT).

Eu vejo assim, para alguns é uma alta no sentido da vida mesmo, de retornar ao trabalho, de sair daqui de dentro e construir uma vida fora daqui (GRDT).

Para alguns eu vejo como não estigmatizar ele como "ele tem transtorno mental, ele vai ficar a vida inteira dele no CAPS". Ele está estável, mas ele não vai dar conta de voltar a trabalhar, mas ele não dá conta de outras 
atividades, mas nem por isso ele precisa ficar dentro do CAPS como era no hospital psiquiátrico! Porque eu vejo que as famílias ainda têm essa dificuldade, e muitos profissionais até, de que simplesmente substituiu o hospital psiquiátrico pelo CAPS. Ele saiu do hospital, mas ele tem que estar dentro do CAPS (GRDT).

Os trabalhadores compreendem que a transferência de cuidados ultrapassa o encaminhamento do usuário a outro serviço, mas se refere à transferência para a vida, para que ele possa retornar às suas atividades cotidianas:

Mas tem uma diferença entre não estar encapsulado e estar desligado do serviço. Porque muitas vezes a alta significa "vamos riscar o sujeito do mapa". Mas ao menos não encapsulado, ele está incluído, ele pode estar incluído, ele está incluído, mas não está encapsulado (GRDT).

Há um posicionamento claro de que o CAPS deve ser transitório na vida do usuário:

O CAPS não é lugar para ficar. Quando o usuário está estabilizado vai para o território. Mudança do termo "alta" - quando fala alta ele acha que está livre do tratamento (DCRD1).

Para que ocorra a transição entre o serviço de saúde mental e a atenção primária é necessário que haja diálogo entre as equipes e a inclusão do usuário nesse processo:

Quando eu fui preparado para a alta aqui, mas lá fora eu fiz acompanhamento no postinho [UBS] e fazia uma ocupação de oficinas no postinho também, até um determinado tempo também. Não foi assim tirar daqui e vai para casa e vai tomar o remédio sozinho. Havia um acompanhamento pelo postinho mais próximo da casa (GRDU).

E para que a transferência de cuidados seja possibilitada é importante que a família esteja engajada nos cuidados:

Eu acho que a gente precisa melhorar muito a questão da inclusão da família, acho que tanto assim no atendimento, no tratamento aqui, e principalmente quando for fazer a alta. A gente percebe que quando tem uma família que fez parte do processo de tratamento aqui, frequentou o CAPS nas reuniões de famílias, então quando você vai fazer esse processo de alta, a família tem uma compreensão da doença, ela consegue perceber isso e isso facilita muito o cuidado dela em casa, vai cuidar da medicação, vai ter alguém que sempre vai estar como cuidador (GRDT).

Então, assim, a participação familiar é fundamental (GRDT).

A gente vê os pacientes que dão alta, que tem sua situação familiar bem redondinha, dificilmente voltam (GRDT). Aqueles que participam do processo terapêutico como um todo, faz uma diferença enorme. Enorme! Dificilmente volta [...] E aqueles que a gente sabe, faz a alta, e quando não tem, passa seis meses e o paciente está de volta aqui. Um ano no máximo! (GRDT).

A emoção é grande quando o paciente dá alta, principalmente como o meu filho que ficou muito tempo. Não dava para dar alta para ele. Não saia da crise. Foi muito difícil, até acertar o medicamento (GRDF).

O meu filho permaneceu oito anos no CAPS. [...] Ele sempre aceitou, a medicação ele toma sempre direitinho [...] e depois de oito anos ele deu alta. Hoje ele está de alta, eu estou muito emocionada. [...] O meu filho já está há um bom tempo em casa, e não precisou mais de internação [...] ele tem terapia lá no postinho [UBS] (GRDF).

De acordo com o GRD dos usuários, um dos fatores impeditivos para a alta é a interrupção do tratamento medicamentoso: 
Uma coisa que talvez impeça a alta é não fazer o tratamento certo, começar a não tomar o remédio [...] Porque se fizer o tratamento certo e se tiver um acompanhamento bom, tanto aqui dentro como dos familiares lá fora, ajuda bastante (GRDU).

Em contraponto, muitas vezes quem demonstra insegurança em promover a transferência de cuidados dos usuários é a equipe do CAPS, pois tem receio de transferi-los:

Acho que eles tem que sair, não acho saudável ficar no CAPS. Quantas vezes acontece, adequou, mas continuou ficando aqui dentro, sem a gente perceber, não é saudável, mas acontece bastante de ficarem anos e anos aqui. Tem familiares que querem, não é um manicômio, não da para fazer disso aqui um "minicômio", eles tem a casa, a gente tem que estar proporcionando isso, esse convívio familiar e não o contrário (DCRD1).

Uma saída apontada pelos trabalhadores para a inserção territorial dos usuários é a criação de um centro de convivência na comunidade. Avalia-se como um importante dispositivo para potencializar as transferências de cuidados e, além disso, extrapola a lógica de encaminhamentos apenas para serviços de saúde.

A gente começa a sentir alguns furos na rede, porque, por exemplo, a gente não tem um centro de convivência, onde eles se sintam um pouco seguros, liberar um pouco aqui do CAPS, mas deixar para o centro de convivência. Mas a gente só tem uma residência terapêutica na cidade, a gente não tem um centro de convivência (GRDT).

Por meio da análise dos resultados avalia-se que a alta dos usuários de CAPS não deve estar atrelada somente ao encaminhamento para outro serviço de saúde, e sim é compreendida como transferência de cuidados, que pode ser feita para a UBS, para outro serviço, para a comunidade e para a vida.

O CAPS deve ser um serviço transitório para os usuários, e não um local de permanência extensa, sendo que no momento em que estão estáveis, devem seguir sua trajetória em outros pontos da rede de saúde e social.

É importante que haja diálogo entre os usuários, seus familiares e equipes de saúde, a fim de que todos estejam engajados no processo de alta e sintam-se seguros quanto aos fluxos no território.

\section{DISCUSSÃO}

A transferência de cuidados constitui-se em uma nova compreensão sobre a alta de usuários de saúde mental. Os participantes deste estudo problematizam essa questão, pois compreendem que ao utilizar o termo "alta" estão remetendo ao sentido de cura. E que a alta no campo da saúde mental ultrapassa essa compreensão, pois os cuidados nesse contexto devem ser longitudinais, em rede e em diferentes serviços de saúde e outras instituições, para além da concepção de que saúde mental somente é contemplada em serviços especializados.

Quando a transferência de cuidados é realizada do CAPS para a atenção primária, é importante que haja diálogo entre as equipes, a fim de que o usuário transite com segurança entre os serviços. A medida que as equipes mantêm a corresponsabilização pelas demandas dos usuários, conhecem suas histórias de vida, 
contexto e rede social, suas fragilidades, necessidades, assim como suas potencialidades, poderão fornecer suporte integral para o usuário, ultrapassando as questões de saúde.

O usuário pode ter receio de ser transferido para outro serviço, pois não se sente seguro quanto ao suprimento de suas necessidades. Um estudo realizado em Dublin, com 150 pacientes psiquiátricos em atendimento em um centro comunitário de saúde mental, aponta que $98 \%$ deles preferiram frequentar um serviço de psiquiatria especializado, mesmo quando estabilizados em seu tratamento. Isso se dá devido ao receio de que não receberiam atenção psiquiátrica adequada, o que pode restringir significativamente a capacidade de equipes de saúde mental especializada para assumir novas referências de cuidados primários ${ }^{(8)}$.

Em um estudo realizado no Japão outro aspecto que indica impedimento para a alta, é a falta de acompanhamento no que se refere à transição da alta hospitalar em psiquiatria para a continuidade de cuidados em saúde mental comunitária, podendo aumentar a probabilidade de recorrência da crise ou readmissão em serviços hospitalares ${ }^{(1)}$.

A transferência de cuidados em saúde mental avança ao setor saúde, ela é uma alta para a vida, quando o usuário poderá escolher onde continuará sua trajetória no território, com possibilidades de retorno as suas atividades cotidianas que foram interrompidas com a experiência da crise. Sendo assim, é importante que tanto os trabalhadores, usuários e seus familiares tenham em vista de que o CAPS é um serviço estratégico para atendimento quando o usuário não está estável em seu quadro clínico, e que nos demais momentos de sua existência, possa transitar em outros lugares, evitando assim a cronificação.

O CAPS precisa ser questionado cotidianamente em relação ao fato de ser um local de inserção, de reabilitação psicossocial e de resgate de autonomia, caso contrário corre o risco de reproduzir no seu interior o mesmo caráter cronificador do hospital psiquiátrico ${ }^{(15)}$.

Há a necessidade, além da compreensão teórica do território, de superar as barreiras da diferença do lugar social habitado pelos indivíduos envolvidos na clínica, tendo o conceito de que esse é um produto de relações sociais das quais os usuários são protagonistas. O CAPS inserido e constituído como um território deve vigiar para não ser um território distinto, à parte ${ }^{(16)}$. Os usuários devem ser o foco de qualquer transferência de cuidados ${ }^{(17)}$.

E ainda, familiares participativos na continuidade do tratamento, comprometidos com o suporte emocional e afetivo dos usuários são parceiros fundamentais para a promoção e manutenção da alta. Porém, um dos desafios para a equipe do CAPS é a constituição de um contrato em que os familiares possam se sentir atores de um projeto de tal maneira que essa adesão envolva a promoção de um espaço de negociação(15)

De acordo com os depoimentos dos participantes, um dos fatores impeditivos para a transferência de cuidados consiste em interromper o tratamento medicamentoso, pelo fato de o usuário sentir-se curado e, portanto, suspender o uso de psicofármacos.

Outro fator impeditivo é a insegurança dos trabalhadores, usuários e familiares em promover e aceitar 
a transferência de cuidados, o que leva o CAPS a ser "minicômio" (pequeno manicômio), como referem os trabalhadores participantes deste estudo.

É importante que a transferência de cuidados promova o vínculo do usuário com outros espaços territoriais: escola, associação de moradores do bairro, espaços de lazer entre outros para que ele possa explorar outras possibilidades de vida.

A concepção de alta em saúde mental, indicada pelos participantes como "transferência de cuidados", é a possibilidade que mais converge para contemplar a subjetividade e integralidade das ações em saúde mental, as quais ultrapassam o setor saúde, e avançam para o território. O termo transferência de cuidados dá a ideia de longitudinalidade, responsabilização e movimento na rede de saúde e rede social dos usuários.

\section{CONCLUSÃO}

A transferência de cuidados é um marcador importante para a consolidação da atenção psicossocial, pois possibilita que os usuários avancem em suas trajetórias na rede de maneira autônoma e cidadã.

Em relação aos encaminhamentos na transferência de cuidados, não é possível formalizar um fluxo de transferência para o território único e uniforme, pois as ações de saída do CAPS devem estar em consonância com a vivência e o momento de cada usuário.

Embora os CAPS sejam estratégicos no cuidado em saúde mental, não devem ser serviços que mantenham os usuários em acompanhamento contínuo por longo período, e sim devem ser transitórios, possibilitando o fortalecimento do cuidado em outros pontos da rede, estreitando vínculo com a atenção primária e com os demais equipamentos sociais do território.

Este estudo aponta como avanço a possibilidade de discutir e problematizar as questões que envolvem a alta dos usuários de saúde mental, compreendida nesse estudo como transferência de cuidados. E também a questão a cronificação dos usuários nos CAPS, quando a alta do serviço não é promovida.

No Brasil há escassez de publicações acerca da temática, sendo necessário que surjam novas pesquisas sobre a alta dos usuários de serviços de saúde mental e as consequências de manter os usuários em acompanhamento contínuo no CAPS, sem dispor de outros dispositivos, o que apontará os limites e desafios para romper a lógica de que saúde mental só pode ser contemplada em serviços especializados, fortalecendo assim a desinstitucionalização e boas práticas em saúde mental.

\section{REFERÊNCIAS}

1. Niimura J, Tanoue M, Nakanishi M. Challenges following discharge from acute psychiatric inpatient care in Japan: patients' perspectives. J Psychiatr Ment Health Nurs [Internet]. 2016 [acesso em: 29 nov. 2017];23(9-10):576-84. Disponível em: http://doi.org/10.1111/jpm.12341.

2. Goldman J, Reeves S, Wu R, Silver I, MacMillan K, Kitto S. A sociological exploration of the tensions related to interprofessional collaboration in acute-care discharge planning. J Interprof Care [Internet]. 2016 [acesso em: 29 nov. 2017];30(2):217-25. Disponível em: http://doi.org/10.3109/13561820.2015.1072803. 
3. Hengartner MP, Klauser M, Heim G, Passalacqua S, Andreae A, Rössler W, et al. Introduction of a Psychosocial PostDischarge Intervention Program Aimed at Reducing Psychiatric Rehospitalization Rates and at Improving Mental Health and Functioning. Perspect Psychiatr Care [Internet]. 2017 [acesso em: 29 nov. 2017];53(1):10-5. Disponível em: http://doi.org/10.1111/ppc.12131.

4. Pang SM, Yeung FK, Cheung EF, Mui J, Chien WT, Leung SF, et al. Health outcomes, community resources for health, and support strategies 12 months after discharge in patients with severe mental illness. Hong Kong Med J [Internet]. 2015 [acesso em: 29 nov. 2017];21 Suppl 2:32-6. Disponível em:

http://www.hkmj.org/abstracts/v21\%20Suppl\%202n/32.htm.

5. Dimenstein M, Bezerra CG. Alta-Assistida de usuários de um hospital psiquiátrico: uma proposta em análise. Physis [Internet]. 2009 [acesso em: 29 nov. 2017];19(3):829-48. Disponível em: http://dx.doi.org/10.1590/S010373312009000300016.

6. Mental Health Commission. Blueprint II: How things need to be [Internet]. Wellington: Mental Health Commission, 2012 [acesso em: 29 nov. 2017]. Disponível em:

http://www.hdc.org.nz/media/207642/blueprint\%20ii\%20how\%20things\%20need\%20to\%20be.pdf.

7. Lin EC, Chan CH, Shao WC, Lin MF, Shiau S, Mueser KT, et al. A randomized controlled trial of an adapted Illness Management and Recovery program for people with schizophrenia awaiting discharge from a psychiatric hospital. Psychiatr Rehabil J [Internet]. 2013 [acesso em: 29 nov. 2017];36(4):243-9. Disponível em: http://doi.org/10.1037/prj0000013.

8. Stangroom R, Morriss M, Soosay I. Patient engagement with primary health care following discharge from community mental health services. N Z Med J [Internet]. 2014 [acesso em: 29 nov. 2017];127(1405):15-23. Disponível em: https://www.nzma.org.nz/journal/read-the-journal/all-issues/2010-2019/2014/vol-127-no-1405/6345.

9. Kantorski LP. CAPSUL I. Avaliação dos Centros de Atenção Psicossocial da Região Sul do Brasil: relatório final. Pelotas: UFPel, 2007.

10. Kantorski LP. CAPSUL II. Avaliação dos Centros de Atenção Psicossocial da Região Sul do Brasil: projeto de pesquisa. Pelotas: UFPel, 2010.

11. Guba E, Lincoln Y. Avaliação de quarta geração. Campinas: Unicamp, 2011.

12. Kantorski LP, Wetzel C, Olschowsky A, Jardim VMR, Bielemann VLM, Schneider JF. Avaliação de quarta geração: contribuições metodológicas para avaliação de serviços de saúde mental. Interface - Comun Saúde, Educ [Internet]. 2009 [acesso em: 29 nov. 2017];13(31):343-55. Disponível em: http://dx.doi.org/10.1590/S1414-32832009000400009. 13. Lincoln Y, Guba E. Naturalistic inquiry. Newbury Park: Sage Publications; 1985.

14. Resolução no 466, de 12 de dezembro de 2012 (BR) [Internet]. Aprova as diretrizes e normas regulamentadoras de pesquisas envolvendo seres humanos. Diário Oficial da União. 12 dez. 2012 [acesso em: 29 nov. 2017]. Disponível em: http://bvsms.saude.gov.br/bvs/saudelegis/cns/2013/res0466_12_12_2012.html.

15. Wetzel C, Kantorski LP, Olschowsky A, Schneider JF, Camatta MW. Dimensões do objeto de trabalho em um Centro de Atenção Psicossocial. Cien Saude Colet [Internet]. 2011 [acesso em: 29 nov. 2017];16(4):2133-43. Disponível em: http://doi.org/10.1590/S1413-81232011000400013.

16. Santos MRP, Nunes MO. Território e saúde mental: um estudo sobre a experiência de usuários de um Centro de Atenção Psicossocial, Salvador, Bahia, Brasil. Interface - Comun Saúde, Educ [Internet]. 2011 [acesso em: 29 nov. 2017];15(38):715-26. Disponível em: http://doi.org/10.1590/S1414-32832011005000036.

17. Sampson R, Cooper J, Barbour R, Polson R, Wilson P. Patients' perspectives on the medical primary-secondary care interface: systematic review and synthesis of qualitative research. BMJ Open [Internet]. 2015 [acesso em: 29 nov. 2017];5(10):e008708. Disponível em: http://doi.org/10.1136/bmjopen-2015-008708. 